\title{
Does mental fatigue impair physical performance? A replication study
}

\author{
Darías Holgado ${ }^{1^{*}}$, Esther Troya ${ }^{1}$, José C. Perales ${ }^{1}$, Miguel A. Vadillo² and Daniel Sana- \\ bria $^{1}$ \\ 1 Centro de Investigación, Mente, Cerebro y Comportamiento, Departamento de Psicología Experimental, Universidad de Gra- \\ nada, España. \\ 2 Departamento de Psicología Básica, Universidad Autónoma de Madrid. España.
}

Objective: to replicate the hypothesis that mental fatigue impairs physical performance.

Design: a pre-registered (https://osf.io/wqkap/), randomized, within-subject experiment.

Methods: 30 recreationally active adults completed a time-to-exhaustion test (TTE) at $80 \% \mathrm{VO}_{2}$ max in two separate sessions, after completing a mental fatigue task or watching a documentary for $90 \mathrm{~min}$. We measured power output, heart rate, (session) RPE and subjective mental fatigue state.

Results: Bayes factor analyses revealed extreme evidence supporting the alternative hypothesis that the mental fatigue task was more mentally fatiguing than the control task, $\mathrm{BF}_{01}=0.009$. However, we found moderate-to-strong evidence for the null hypothesis (i.e., no evidence of reduced performance) for average time in TTE $\left(\mathrm{BF}_{01}=9.762\right)$ and anecdotal evidence for the null hypothesis in (session) RPE $\left(\mathrm{BF}_{01}\right.$ $=2.902)$ and heart rate $\left(\mathrm{BF}_{01}=2.587\right)$.

Conclusions: our data seem to challenge the idea that mental fatigue has a negative influence on exercise performance. Although we did succeed at manipulating subjective mental fatigue, this did not impair physical performance. However, we cannot discard the possibility that mental fatigue may have a negative influence under conditions not explored here, e.g., individualizing mentally fatiguing tasks. In sum, further research is warranted to determine the role of mental fatigue on exercise and sport performance. 


\section{Introduction}

Recent research suggests that cognitive processes, such as inhibitory or cognitive control, are fundamental to achieve a high level of performance in sports (Vickers \& Williams, 2017). Under this assumption, a sizable volume of literature has explored the theme of the potential negative influence of mental fatigue on subsequent exercise (Pageaux \& Lepers, 2018). Mental fatigue is considered a complex psychobiological state induced by a prolonged period of demanding cognitive activity, which usually has a negative impact on cognitive function (Ishii et al., 2014). In addition, mental fatigue may affect several objective and subjective measures, such as feeling of tiredness and lack of energy (Boksem \& Tops, 2008), increased perception of effort (Van Cutsem et al., 2017), altered brain activity (Wang et al., 2016), or decreased response accuracy (Boksem \& Tops, 2008).

To examine whether mental fatigue has a negative effect on exercise performance, Marcora et al. (2009) asked participants $(N=16)$ to carry out a demanding cognitive task (experimental condition) or watch a documentary (control condition) for 90 minutes before a cycling time-to-exhaustion test at $80 \%$ of their peak power output. The AX-continuous performance task (AX-CPT) was chosen to induce mental fatigue because it requires sustained attention, working memory, response inhibition, and error monitoring (Wiemers \& Redick, 2018). The results of the study showed that, in the mental fatigue condition, participants reached exhaustion sooner than in the control condition (Marcora et al., 2009). The starting hypothesis of the study was that mental fatigue increases rating of perceived exertion (RPE) during exercise, and consequently athletes would fatigue sooner (Pageaux, 2014; Van Cutsem et al., 2017). As predicted, RPE was influenced by the mental fatigue manipulation, but motivation to exercise did not change. This result is in line with the psychobiological model of endurance performance that suggests that perception of effort during exercise is a key variable for exercise regulation. Therefore, a higher mental fatigue might lead to a higher RPE which would subsequently impair endurance performance (Pageaux, 2014).

Marcora et al.'s (2009) report was the first of a series of studies looking at the negative influence of mental fatigue on exercise performance. However, a closer inspection of a recent review (Pageaux \& Lepers, 2018) reveals that almost 50 percent of the studies in this literature showed no effects of mental fatigue on exercise performance. Likewise, most of these studies have relied on small samples (mean $N=13$, with an 8-25 range), which renders them underpowered to detect small but non trivial effect sizes. Somewhat paradoxically, low power can also increase the probability of false positives (Button et al., 2013). Furthermore, the evidence for this relationship is inconclusive, as subsequent studies did not confirm that mental fatigue affected RPE, even when exercise performance did decrease (Martin et al., 2016; Penna et al., 2018).

In sum, we believe that current evidence is inconclusive, at best, regarding the potential effect of mental fatigue (induced by a demanding cognitive task) on exercise performance and perceived exertion. This 
is a topic of interest for athletes and trainers, to the extent that the potential adverse impact of mental fatigue on exercise could justify the necessity to implement training strategies to counteract the negative consequences of mental fatigue (Russell et al., 2019). In addition, research on mental fatigue and exercise performance bears some resemblance to research conducted in the controversial domain of ego depletion (Baumeister et al., 1998), which has been challenged in recent years by an increasing number of failed replications (Carter et al., 2015; Hagger et al., 2016; Vadillo, 2019). Therefore, we consider pertinent to replicate Marcora et al.'s (2009) original study to investigate the reliability of their findings.

\section{Methods}

\section{Design}

The experiment followed a pre-registered protocol (https://osf.io/7hz3x), with a single-blind, counterbalanced, within-participant design. This study was approved by the University of Granada Ethics Committee (287/CEIH/2017). All experimental procedures complied with the Declaration of Helsinki. Before being recruited for the study, participants read and signed an informed consent. All data were entered in a case report form and subsequently in a computerized database. Participants were naïve to the aim of the study in order to avoid expectation effects. Once they completed their participation, they were debriefed with the purpose of the study.

\section{Participants}

We recruited participants from the Granada area population, in Spain. Experimental sessions took place in the Mind, Brain, Behaviour Research Centre, at the University of Granada. We recruited male and female recreationally active adults involved in regular aerobic training $(>6 \mathrm{~h} /$ week), with ages between 18 and 44, free of any known disease, and not taking any medication with the exception of contraceptives. Exclusion criteria were the presence of symptomatic cardiomyopathy, metabolic disorders such as obesity (BMI $>30)$ or diabetes, chronic obstructive pulmonary disease, epilepsy, therapy with $\beta-$ blockers and medications that would alter cardiovascular function, hormonal therapy, smoking, and neurological disorders.

Sample size was determined by using sequential testing with one-sided Bayes factors. We planned to collect data from a minimum of 30 participants and then monitor the Bayes factor for the average time in the time-to-exhaustion test until it reached moderate evidence in favor of either the alternative $\left(\mathrm{BF}_{01}\right.$ $<1 / 6)$ or the null hypothesis $\left(\mathrm{BF}_{01}>6\right)$. The alternative hypothesis was that performance in the mental fatigue condition would be reduced relative to the control condition (i.e., $\mathrm{MF}<\mathrm{CON}$ in time to exhaustion, and MF > CON in RPE), so the null hypothesis was the non-existence of a detrimental effect of the mental fatigue manipulation on exercise performance. We used a half-Cauchy prior with width 0.707 (the default width in JASP (JASP Team, 2019)). If none of these thresholds was reached, we 
planned to terminate data collection if the sample size reached 50 participants or if none of the previous criteria was met by July, 2019. A final sample of 30 ( 24 males, 6 females) participants with an age 23.5 \pm 6.3 years, $\mathrm{VO}_{2} \mathrm{Max} 41.88 \pm 9.08 \mathrm{mil} / \mathrm{min} / \mathrm{kg}$, completed the study.

\section{Apparatus and materials}

We used a ViaSprint 150P cycle ergometer (Ergoline GmbH, Germany) to induce physical effort and to obtain power values, and a JAEGER Master Screen gas analyser (CareFusion GmbH, Germany) to provide a measure of gas exchange during the effort test. We used a V800 Polar monitor (Polar Electro, Finland) to monitor and record (through a Polar H7 sensor band attached to the participant's chest) the heart rate (HR) of the participants during the experiments. We used a PC and the E-Prime software (Psychology Software Tools, Pittsburgh, PA, USA) to control the stimulus presentation and response collection for the AX-CPT task. Moreover, we used the same PC screen to reproduce the documentary in the control condition.

\section{Procedure}

\section{Screening visit}

During the first visit, participants performed an incremental exercise test until exhaustion. The test began with a load of $50 \mathrm{~W}$ for 2 minutes and then the load incremented automatically in steps of $50 \mathrm{~W}$ every 2 minutes until volitional exhaustion (i.e., a pedal frequency of less than $60 \mathrm{rpm}$ for more than 5 seconds despite strong verbal encouragement). All participants reached their peak effort in the incremental test, according to the respiratory exchange ratio values (RER $\geq 1.1$ ). We used the $80 \%$ of the peak power output in this session to establish the intensity of the time-to-exhaustion test. This value was determined by the last step completed or the fraction of the remaining time to complete that steep. During this first visit to the lab, participants were also provided with standardized written instructions (Pageaux, 2016) to familiarize with the RPE Borg's scale (Borg, 1982).

\section{Experimental session}

After the familiarization visit, participants attended to the lab on two separate sessions to perform either the AX-CPT task or watch the documentaries before the cycling time-to-exhaustion test. Upon arrival, participants filled in the Brunel Mood Scale (BRUMS) to assess mood and a VAS to assess the task demands (see subjective scales below). After that, they completed the treatment in a counterbalanced order. Once participants had completed the task, they filled in again the BRUMS, and the VAS, together with a motivation questionnaire. Immediately afterwards, they were positioned on the cycle ergometer to start the time-to-exhaustion test approximately 15 minutes after the end of treatment. This constantpower cycling test can detect changes in endurance performance (Amann et al., 2008). The time-to 
exhaustion cycling test consisted of a 3-min warm-up at $40 \%$ of peak power output followed by a rectangular workload corresponding to $80 \%$ of peak power output achieved in the screening visit. The test finished when the pedal frequency was less than 60 RPM for more than $5 \mathrm{~s}$ despite standardized verbal encouragement. To increase participants' motivation, we offered a prize of $50 €$ for the best cycling performance after each treatment. The third visit was exactly the same except for the treatment, which was the opposite of the second visit.

\section{Mental fatigue condition}

In the mental fatigue condition, participants completed a 90 minutes long AX-CPT (Barch et al., 1997). Participants seated in a room in front of a 21' PC screen, and sequences of letters were visually presented one at a time in a continuous fashion. They were instructed to press the right button on target trials and the left button otherwise. Target trials were defined as a cue-probe sequence in which the letter A appeared as the cue and the letter $\mathrm{X}$ appeared as the probe. The remaining letters of the alphabet served as invalid cues and nontarget probes, with the exception of the letters $\mathrm{K}$ and $\mathrm{Y}$, which were excluded because of their similarity in appearance to the letter X. Letter sequences were presented in pseudorandom order, such that target (AX) trials occurred with $70 \%$ frequency and nontarget trials occurred with $30 \%$ frequency. Non-targets were divided evenly (10\% each) among the following trial types: BX trials, in which an invalid cue (i.e., non-A) preceded the target; AY trials, in which a valid cue followed by a non-target probe (i.e., non-X); and BY trials, in which an invalid cue followed by a non-target probe. To increase task difficulty, two white distractor letters (which could be any letter but A, K, X, or Y) were presented between the cue and probe, which were both red. All letters were presented centrally, on a black background, for a duration of $300 \mathrm{~ms}$ in 24-point uppercase Courier font. Each letter was followed by a 1,200-ms interval, which gives a 4,500-ms delay between the presentation of cue and probe stimuli. Any missed or incorrect response elicited a bleep sound from two speakers as a prompt to increase speed and accuracy. To further increase engagement in the AX-CPT, a $50 €$ prize was given for the best performance. Feedback on performance was presented on the computer screen every $30 \mathrm{~min}$ as a percentage of the maximum possible score. Performance was scored automatically by the computer on the basis of correct responses and response times. Because a reduction in vigilance and working memory are well-established effects of mental fatigue, the proportion of correct responses to the AX trials during the first and last 15-min period of the AX-CPT was compared as a manipulation check.

\section{Control condition}

In the control condition, participants watched two documentaries (approximately 45 minutes each), which were able to maintain a neutral mood, during 90 minutes in the same PC screen used for the AXCPT. These two documentaries were the Spanish version of "World Class Trains-The Venice Simplon 
Orient Express" (Pegasus-Eagle Rock Entertainment, 2004) and "The History of Ferrari-The Definitive Story" (Boulevard Entertainment, 2006).

\section{Subjective scales}

Rating of perceived exertion (RPE): we asked participants to rate their perceived effort in the 6-20 RPE (Borg, 1982) scale after the time-to-exhaustion test (approximately 10 minutes) and after reading a standardized written instruction (Pageaux, 2016).

BRUMS. We assessed participants' mood before and after both treatments with The Brunel Mood Scale (BRUMS) developed by Terry et al. (2003). The questionnaire is based on Profile of Mood States, but it only contains 24 items (e.g., angry, uncertain, miserable, tired, nervous, energetic) divided into six respective subscales: anger, confusion, depression, fatigue, tension, and vigor. The items are answered on a 5-point Likert scale ( 0 not at all, 1 a little, 2 moderately, 3 quite a bit, 4 extremely), and each subscale, with four relevant items, yields a raw score in the range of 0 to 16 . We only considered the subscales of fatigue and vigor as the main items to assess the state of mental fatigue.

VAS. We used a visual analog scale (VAS) to check the task demands of mental fatigue and control condition to the following questions: 1) What is your activation level now?" 2) "What is your physical fatigue level now?" and 3) "What is your mental fatigue level now?" (Holgado et al., 2018)

Motivation. We assessed motivation before the time-to-exhaustion tests with the success motivation and intrinsic motivation scales developed and validated by Matthews et al. (2001). Each scale consists of 7 items (e.g., "I want to succeed on the task" and "I am concerned about not doing as well as I can") scored on a 5-point Likert scale ( 0 not at all, 1 a little bit, 2 somewhat, 3 very much, 4 extremely). The final score of the questionnaire ranged between 0 and 28 .

\section{Statistical analysis}

We calculated one-sided Bayes factors for paired-samples $t$-tests (mental fatigue vs control) using the open-source JASP statistical package (JASP Team, 2019). The alternative hypothesis was the existence of a reduced performance in the mental fatigue condition compared to the control condition ( $\mathrm{MF}<\mathrm{CON}$ in time to exhaustion, and MF > CON in RPE), so the null hypothesis was the non-existence of a detrimental effect of the mental fatigue manipulation on exercise performance. Additionally, we used a onesided test for each pairwise comparison (e.g., mental fatigue condition would reduce motivation or increase the subjective mental fatigue state compared to control). As prior distribution of the population effect size $(\delta)$, we used a zero-truncated Cauchy distribution with 0.707 width. To ensure that this arbitrary choice did not affect the results, we conducted robustness checks with a wide range of alternative scaling factors (see supplemental materials). 
The dependent variables for the time-to-exhaustion test were the average time completed by participants, the average heart rate and the session RPE.

\section{Manipulation check}

The psychological variables for the mental fatigue or control conditions were the BRUMS values for fatigue and vigor, the upcoming motivation to performance physical task and the VAS. These data scales were analyzed by (normalized) rating change: post-test rating minus pre-test rating divided by post-test rating plus pre-test rating.

\section{Results}

\section{Confirmatory analysis}

\section{AX-CPT task}

The one-sided Bayes factor for the accuracy in the AX-CPT task between the first and the last 15 minutes of the task was $\mathrm{BF}_{01}=4.04$, indicating that the observed data support the null hypothesis of no effect. According to the interpretation guidelines proposed by Jeffrey (Jeffreys, 1961), this represents moderate evidence for the null hypothesis that there is no decline in accuracy between the first and the last 15 minutes. The accuracy (percentage of correct responses) for both periods were: 0.953 (95\% Credible Interval (CI) 0.932 - 0.975) and 0.95 (95\% CI 0.934 - 0.966) for the first and last 15 minutes, respectively.

\section{Subjective scales}

The one-sided Bayes factor for the normalized VAS score for the question "1) What is your activation level now?" was $\mathrm{BF}_{01}=0.69$, which represents anecdotal evidence in favor of the alternative hypothesis. The normalized VAS score for both conditions were: -0.17 (95\%CI $-0.271--0.068$ ) IU and -0.296 (95\%CI -0.43 - -0.179) IU, for the control and mental fatigue conditions, respectively.

The one-sided Bayes factor for the normalized VAS score for "What is your physical fatigue level now?" was $\mathrm{BF}_{01}=0.136$, providing substantial support for the alternative hypothesis that fatigue increased after the task. The normalized VAS scores for both conditions were: -0.148 (95\%CI -0.261 - $0.034) \mathrm{IU}$ and $-0.17(95 \% \mathrm{CI}-0.068-0.268)$ IU for the control and mental fatigue conditions, respectively.

The one-sided Bayes factor for the normalized VAS score for "What is your mental fatigue level now?" (Fig. 1) was $\mathrm{BF}_{01}=0.009$, which represents extreme evidence in favor of the alternative hypothesis, i.e., that the mental fatigue task was more mentally fatiguing than the control task. The normalized VAS scores for both conditions were: 0.08 (95\%CI -0.061 - 0.221) IU and 0.371 (95\%CI 0.251 - 0.492) IU for the control and mental fatigue conditions, respectively. 
The one-sided Bayes factor for the normalized VAS score for "What is your mental fatigue level now?" (Fig.1) was $\mathrm{BF}_{01}=0.009$, which represents extreme evidence in favor of the alternative hypothesis, i.e., that the mental fatigue task was more mentally fatiguing than the control task. The normalized VAS scores for both conditions were: 0.08 (95\%CI -0.061 - 0.221) IU and 0.371 (95\%CI 0.251 - 0.492) IU for the control and mental fatigue conditions, respectively.

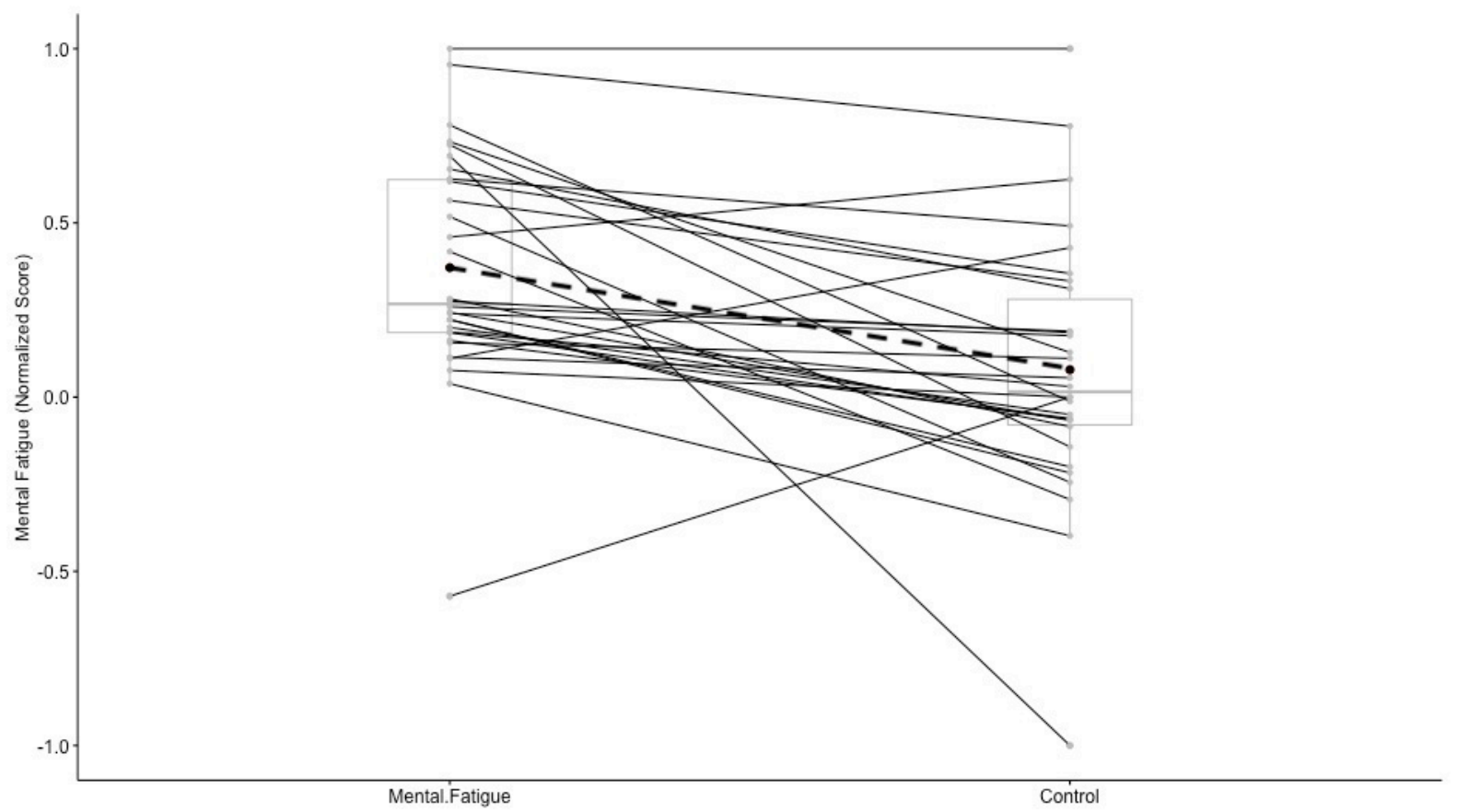

Fig 1. Normalized VAS score for each participant and condition for the question "What is your mental fatigue level now?". Dashed line represents the overall mean.

The one-sided Bayes factor for the BRUMS score of fatigue was $\mathrm{BF}_{01}=0.025$, providing very strong evidence in favor of the alternative hypothesis. The normalized score for both conditions were: 0.106 $(95 \% \mathrm{CI}-0.058-0.270) \mathrm{IU}$ and $0.442(95 \% \mathrm{CI} 0.312-0.572)$ IU for the control and mental fatigue conditions. Likewise, the one-sided Bayes factor for the BRUMS score of vigor was $\mathrm{BF}_{01}=0.306$, This provides moderate evidence in support of the alternative hypothesis. The normalized score for both conditions were: $-0.62(95 \% \mathrm{CI}-0.174-0.05)$ IU and -0.269 (95\% CI $-0.416--0.123)$ IU for the control and mental fatigue conditions, respectively.

Contrary to the initial hypothesis, the one-sided Bayes factor for the motivation score was $\mathrm{BF}_{01}=8.287$, which provides moderate evidence in favour of the null hypothesis, i.e., mental fatigue did not have a detrimental effect on motivation. The scores for the two conditions were: 13.07 (95\%CI 11.73 - 14.41) IU and 13.53 (95\%CI 11.85 - 15.21) IU for the control and mental fatigue condition, respectively.

\section{Performance}

The one-sided Bayes factor for the time-to-exhaustion (Fig.2) measure was $\mathrm{BF}_{01}=9.762$, indicating that the observed data moderately to strongly support the null hypothesis that mental fatigue did not 
have a detrimental effect on exercise performance. The average time completed for both conditions was: 765.5 (SE 92.05, 95\%CI 577.2 - 953.8) and 705.4 (SE 79.49, 95\%CI 542.8 - 868) seconds for the mental fatigue and control conditions, respectively.

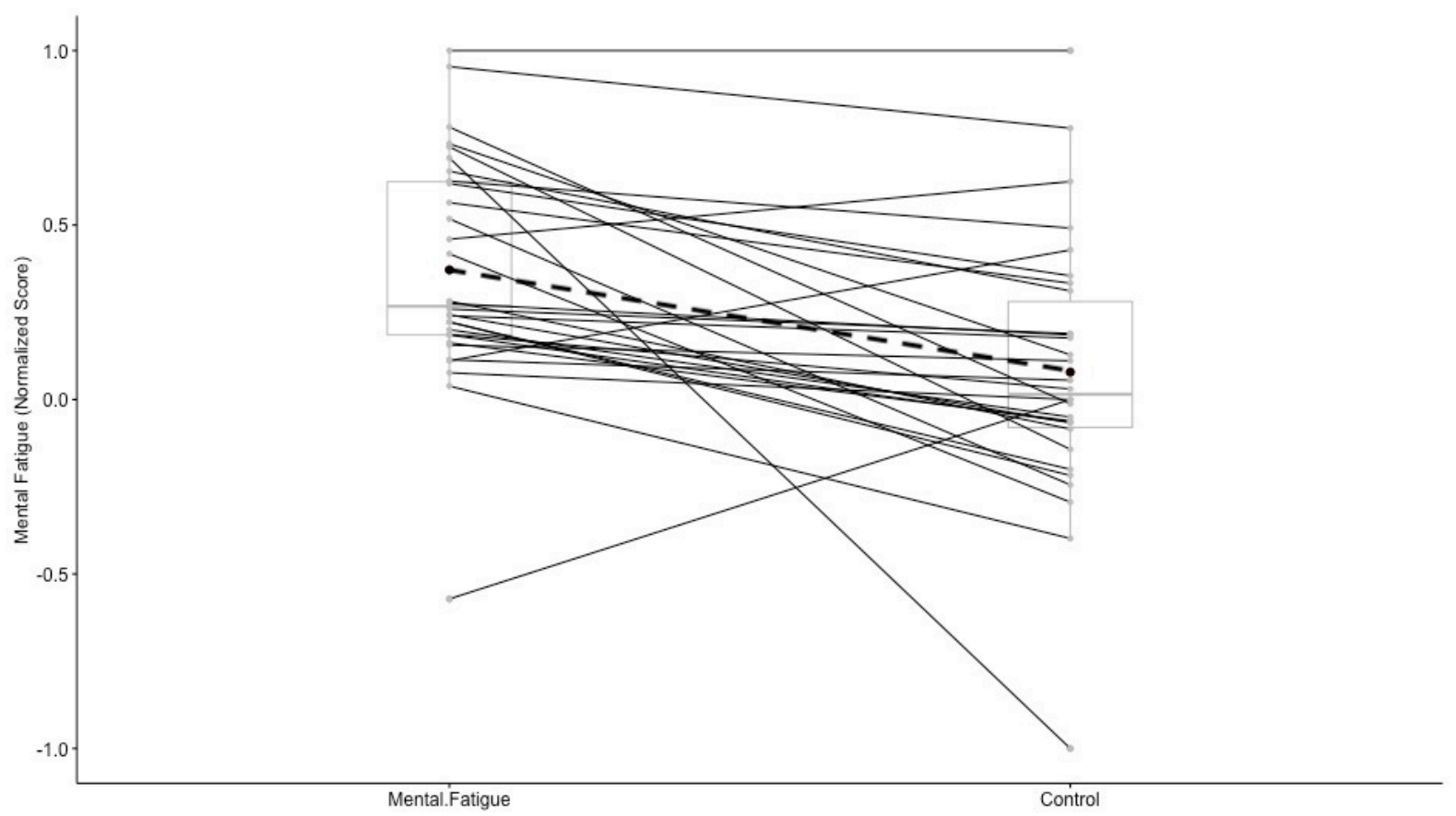

Fig 2. Time-to-exhaustion (seconds) for each participant and condition during the $80 \%$ cycling time-to-exhaustion test. Dashed line represents the overall mean.

The one-sided Bayes factor for the average heart rate during cycling time-to-exhaustion test was $\mathrm{BF}_{01}$ $=2.587$, indicating anecdotal evidence in favor of the null hypothesis that mental fatigue did not have a detrimental effect on the average heart rate. The average heart rate for both conditions was: 161 (95\%CI 159 - 165) bpm and 156 (95\%CI 156 - 165) bpm for the control and mental fatigue conditions, respectively.

The one-sided Bayes factor for the average session RPE (Fig.3) after the cycling time-to-exhaustion was $\mathrm{BF}_{01}=2.902$, which indicates anecdotal evidence in favor of the null hypothesis that mental fatigue did not have a detrimental effect on RPE. The average RPE for both conditions was: 15.8 (95\%CI 14.94 -16.66) IU and 15.93 (95\%CI 15.08 - 16.79) IU for the control and mental fatigue conditions, respectively. 


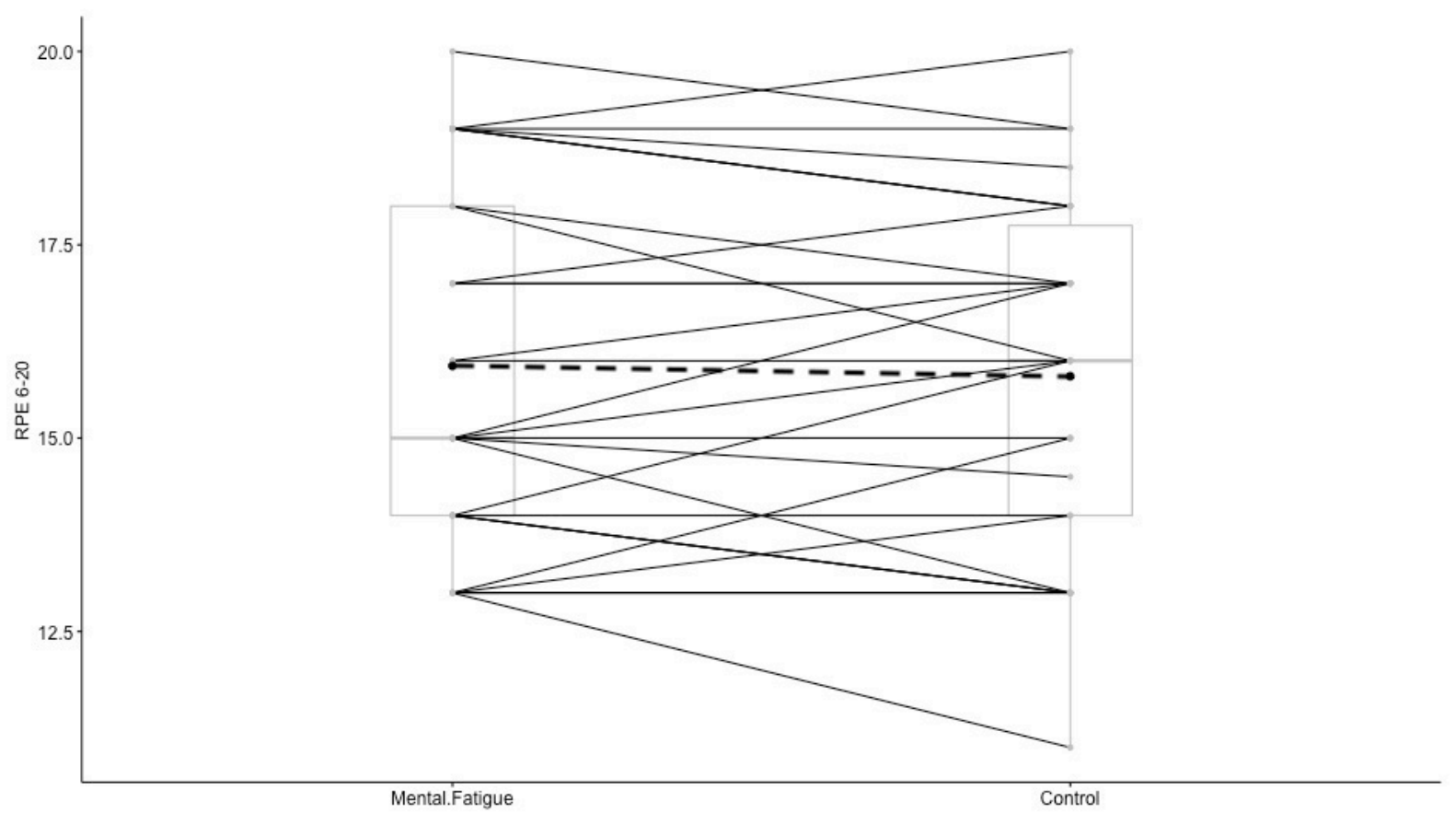

Fig 3. RPE for each participant and condition after the $80 \%$ cycling time-to-exhaustion test. Dashed line represents the overall mean.

\section{Discussion}

The purpose of this study was to replicate the effect of mental fatigue on physical performance in humans reported by Marcora et al. (2009). The results showed that even though participants felt more mentally fatigued in the mental fatigue condition (AX-CPT session), we did not find evidence of an inferior physical performance in the mental fatigue condition. No evidence of increments in RPE and HR or reduction in motivation was found either. Our results are in contrast with those of Marcora et al. (2009) and add to the growing number of studies failing to find any effects of mental fatigue on physical performance (Filipas et al., 2018; Silva-Cavalcante et al., 2018). As pointed out in the Introduction, a recent review (Pageaux \& Lepers, 2018) showed that almost 50 percent of the studies found no effects of mental fatigue on variables related to exercise performance.

Our results cannot definitely discard the existence of a small mental fatigue effect on performance. As shown in robustness analyses, with sufficiently small prior widths, Bayes Factors fall in the anecdotal evidence range (i.e., the available evidence becomes insufficient to choose between the two hypotheses, as the alternative and the null hypotheses grow closer to each other). Beyond the discussion regarding the practical significance of such small effects, their detection would require sample sizes much larger to the ones used to date. Still, as far as we know, we have tested the largest sample of participants in this literature and the results showed that mental fatigue did not influence negatively physical performance, at least to the extent considered in previous studies. If anything, the visual difference between the two conditions was in the direction opposite to the one reported by Marcora et al., i.e., on average, 
participants were exhausted later in the mental fatigue condition than in the control condition (please note that the possibility of a difference in that direction cannot be tested with the one-sided BFs used in the present study).

The results of our study fail to support the assumption of the psychobiological model of endurance performance that mental fatigue would lead to a higher RPE and, consequently, impair exercise performance (Pageaux, 2014; Van Cutsem et al., 2017). Note, though, that compared to the Marcora et al., (2009) we did not measure RPE during the protocol, but the session RPE, thus we do not have data to analyze whether RPE increased over time in the time-to-exhaustion test. Nonetheless, the session RPE is a valid and reliable tool to monitor training load (Haddad et al., 2017). However, even if participants were familiarized with the RPE scale and provided with written instructions, the RPE was assessed approximately 10 minutes after exercise which might have resulted in participants indicating a lower than normal RPE for a time-to-exhaustion test (Fig. 3). This could be considered as a potential limitation of our study. In any case, whatever the reason for those lower than expected RPE values, the mental load manipulation did not seem to exert an effect on either the subjective of objective indexes of performance.

In light of the evidence, some authors have argued that this research area would benefit from adopting a new perspective to explain the phenomenon of fatigue. Kluger et al. (2013) propose that fatigue should be understood within the interaction between performance fatigability and perceived fatigability. Perceived fatigability is derived from the sensations that regulate the integrity of the participant with the aim of maintaining homeostasis and the psychological state (Enoka \& Duchateau, 2016). In that sense, less fatigable individuals would be able to tolerate higher demands to reach a given level of fatigue (Enoka \& Duchateau, 2016). Therefore, rather than long cognitive tasks like the AX-CPT, which might induce sleepiness, future studies should seek to individualize the cognitive load to successfully induce the state of mental fatigue. For example, the study by O'Keeffe et al., (2020) showed that 16 minutes of individualized dual-task based on previous performance was effective to induce a high level of mental fatigue and arousal, whereas the AX-CPT caused a state of mental fatigue, but together with sleepiness and low levels of arousal. Similarly, the control condition in these studies might fail to keep a neutral emotional state, since participants could find the documentaries boring (O'Keeffe et al., 2020).

Another possible explanation for the lack of evidence for the alternative hypothesis in our study could be that an acute session is not effective enough to affect exercise performance and/or that the effects might depend on individual differences. Indeed, we cannot discard the possibility that mental fatigue induced over consecutive days might have a negative influence on performance, as the long-term effects of mental fatigue on exercise performance have not been studied yet (Russell et al., 2019). Moreover, given that almost half of the participants became exhausted sooner in the mental fatigue condition than in the control condition, the inter-individual variability might be an important factor to consider in this 
context. However, supplementary analyses did not show that subjective feeling of mental fatigue correlated with performance impairment (see supplementary materials).

In a broader sense, and going beyond the specific purpose of this replication, we argue that the present data also seem to be in disagreement with a theoretical framework that highlights the role of (top-down) self-control on exercise performance (Dietrich \& Audiffren, 2011; Englert, 2016; Taylor et al., 2018). According to the Reticular-Activating Hypofrontality model, humans have a limited pool of cognitive and metabolic resources and when these resources are shared or depleted, interference between them occurs (Dietrich \& Audiffren, 2011). Therefore, acts of self-control before an exercise would diminish the ability of athletes to self-regulate adequately during exercise (Taylor et al., 2018). In this sense, our study has a certain resemblance of the study of the ego-depletion effect (Baumeister et al., 1998; Hagger et al., 2016). Although this theory has been the foundation of the study of self-control for decades, the reliability of the ego depletion effect has been questioned recently (e.g., Hagger et al., 2016; Vadillo, 2019). Similarly, our data question the existence of an ego-depletion-like effect in the domain of exercise performance.

In conclusion, our data seem to challenge the idea that mental fatigue has a negative influence on exercise performance. Our study, however, does not provide the definitive answer on whether mental fatigue might affect exercise performance under different conditions, but opens new avenues for future research on this topic that should consider factors like individualizing mentally fatiguing tasks or mental fatigue over consecutive days.

\section{Compliance with ethical standards}

Contributors: All authors have made substantial contributions to various elements of the study.

Conceptualization: Darías Holgado, Daniel Sanabria

Data collection: Darías Holgado, Esther Troya

Data curation: Darías Holgado, Esther Troya

Formal analysis: Darías Holgado

Methodology: Darías Holgado, Daniel Sanabria, Jose C. Perales

Project administration: Daniel Sanabria, Darías Holgado, José C. Perales, Miguel Á. Vadillo

Supervision: Daniel Sanabria, José C. Perales, Miguel Á. Vadillo

Writing - original draft: Darías Holgado

Writing - review \& editing: Daniel Sanabria, José C. Perales, Miguel Á. Vadillo 


\section{Funding}

Daniel Sanabria is awarded with a grant PSI2016-75956-P from Ministerio de Economía, Industria y Competitividad, Miguel A. Vadillo is awarded with grants 2016-T1/SOC-1395, from Comunidad de Madrid (Programa de Atracción de Talento Investigador, 2016) and PSI2017-85159-P from Ministerio de Ciencia, Innovación y Universidades. José C. Perales' research is supported by a grant PSI201785488-P from the Ministerio de Economía y Competitividad, co-funded by the Fondo Europeo de Desarrollo Regional, FEDER, European Commission. The funders were not involved directly in the preparation of this manuscript.

\section{Data availability}

Data from the experiment can be found here: https://osf.io/wqkap/

\section{References}

Marcora, S. M. (2008). Similar sensitivity of time to exhaustion and time-trial time to changes in endurance. Medicine and Science in Sports and Exercise, 40(3), 574-578. https://doi.org/10.1249/MSS.0b013e31815e728f

Barch, D. M., Braver, T. S., Nystrom, L. E., Forman, S. D., Noll, D. C., \& Cohen, J. D. (1997). Dissociating working memory from task difficulty in human prefrontal cortex. Neuropsychologia, 35(10), 1373-1380.

Baumeister, R. F., Bratslavsky, E., Muraven, M., \& Tice, D. M. (1998). Ego depletion: Is the active self a limited resource? Journal of Personality and Social Psychology, 74(5), 1252-1265.

Boksem, M., \& Tops, M. (2008). Mental fatigue: Costs and benefits. Brain Research Reviews, 59(1), 125-139. https://doi.org/10.1016/j.brainresrev.2008.07.001

Borg, G. (1982). Psychophysical bases of perceived exertion. Medicine \& Science in Sports \& Exercise, 14(5), 377-381. https://doi.org/10.1249/00005768-198205000-00012

Carter, E. C., Kofler, L. M., Forster, D. E., \& McCullough, M. E. (2015). A series of meta-analytic tests of the depletion effect: Self-control does not seem to rely on a limited resource. Journal of Experimental Psychology. General, 144(4), 796-815. https://doi.org/10.1037/xge0000083 
Dietrich, A., \& Audiffren, M. (2011). The reticular-activating hypofrontality (RAH) model of acute exercise. Neuroscience \& Biobehavioral Reviews, 35(6), 1305-1325. https://doi.org/10.1016/j.neubiorev.2011.02.001

Englert, C. (2016). The strength model of self-control in sport and exercise psychology. Frontiers in Psychology, 7(MAR). Scopus. https://doi.org/10.3389/fpsyg.2016.00314

Enoka, R. M., \& Duchateau, J. (2016). Translating Fatigue to Human Performance. Medicine and Sci$\begin{array}{llll}\text { ence in } & \text { Sports }\end{array}$ https://doi.org/10.1249/MSS.0000000000000929

Filipas, L., Mottola, F., Tagliabue, G., \& La Torre, A. (2018). The effect of mentally demanding cognitive tasks on rowing performance in young athletes. Psychology of Sport and Exercise, 39, 52-62. https://doi.org/10.1016/j.psychsport.2018.08.002

Hagger, M. S., Chatzisarantis, N. L. D., Alberts, H., Anggono, C. O., Batailler, C., Birt, A. R., Brand, R., Brandt, M. J., Brewer, G., Bruyneel, S., Calvillo, D. P., Campbell, W. K., Cannon, P. R., Carlucci, M., Carruth, N. P., Cheung, T., Crowell, A., De Ridder, D. T. D., Dewitte, S., ... Zwienenberg, M. (2016). A Multilab Preregistered Replication of the Ego-Depletion Effect. Perspectives on Psychological Science: A Journal of the Association for Psychological Science, 11(4), 546-573. https://doi.org/10.1177/1745691616652873

Holgado, D., Zandonai, T., Zabala, M., Hopker, J., Perakakis, P., Luque-Casado, A., Ciria, L., GuerraHernandez, E., \& Sanabria, D. (2018). Tramadol effects on physical performance and sustained attention during a 20-min indoor cycling time-trial: A randomised controlled trial. Journal of Science and Medicine in Sport, 21(7), 654-660. https://doi.org/10.1016/j.jsams.2017.10.032

Ishii, A., Tanaka, M., \& Watanabe, Y. (2014). Neural mechanisms of mental fatigue. Reviews in the Neurosciences, 25(4), 469-479. https://doi.org/10.1515/revneuro-2014-0028

JASP Team. (2019). JASP (Version 0.10.2). 
Jeffreys, H. (1961). The Theory of Probability. (3rd ed.). Oxford Univ. Press.

Kluger, B. M., Krupp, L. B., \& Enoka, R. M. (2013). Fatigue and fatigability in neurologic illnesses. Neurology, 80(4), 409-416. https://doi.org/10.1212/WNL.0b013e31827f07be

Marcora, S, Staiano, W., \& Manning, V. (2009). Mental fatigue impairs physical performance in humans. Journal of Applied Physiology, 106(3), 857-864. https://doi.org/10.1152/japplphysiol.91324.2008

Martin, K., Staiano, W., Menaspà, P., Hennessey, T., Marcora, S., Keegan, R., Thompson, K. G., Martin, D., Halson, S., \& Rattray, B. (2016). Superior inhibitory control and resistance to mental fatigue in professional road cyclists. PLoS ONE, 11(7). Scopus. https://doi.org/10.1371/journal.pone.0159907

Matthews, G., Campbell, S. E., \& Falconer, S. (2001). Assessment of Motivational States in Performance Environments. Proceedings of the Human Factors and Ergonomics Society Annual Meeting, 45(13), 906-910. https://doi.org/10.1177/154193120104501302

O’Keeffe, K., Hodder, S., \& Lloyd, A. (2020). A comparison of methods used for inducing mental fatigue in performance research: Individualised, dual-task and short duration cognitive tests are most effective. Ergonomics, 63(1), 1-12. https://doi.org/10.1080/00140139.2019.1687940

Pageaux, B. (2014). The psychobiological model of endurance performance: An effort-based decisionmaking theory to explain self-paced endurance performance. Sports Medicine (Auckland, N.Z.), $1319-1320$.

Pageaux, B. (2016). Perception of effort in Exercise Science: Definition, measurement and perspectives. European Journal of Sport Science, 16(8), 1-10. https://doi.org/10.1080/17461391.2016.1188992

Pageaux, B, \& Lepers, R. (2018). Chapter 16-The effects of mental fatigue on sport-related performance. In Samuele Marcora \& M. Sarkar (Eds.), Progress in Brain Research (Vol. 240, pp. 
291-315). Elsevier. https://doi.org/10.1016/bs.pbr.2018.10.004

Penna, E. M., Filho, E., Wanner, S. P., Campos, B. T., Quinan, G. R., Mendes, T. T., Smith, M. R., \& Prado, L. S. (2018). Mental Fatigue Impairs Physical Performance in Young Swimmers. Pediatric Exercise Science, 30(2), 208-215. https://doi.org/10.1123/pes.2017-0128

Russell, S., Jenkins, D., Smith, M., Halson, S., \& Kelly, V. (2019). The application of mental fatigue research to elite team sport performance: New perspectives [Article in Press]. Scopus. https://doi.org/10.1016/j.jsams.2018.12.008

Russell, S, Jenkins, D., Rynne, S., Halson, S. L., \& Kelly, V. (2019). What is mental fatigue in elite sport? Perceptions from athletes and staff. European Journal of Sport Science, 1-10. https://doi.org/10.1080/17461391.2019.1618397

Silva-Cavalcante, M. D., Couto, P. G., Azevedo, R. de A., Silva, R. G., Coelho, D. B., Lima-Silva, A. E., \& Bertuzzi, R. (2018). Mental fatigue does not alter performance or neuromuscular fatigue development during self-paced exercise in recreationally trained cyclists. European Journal of Applied Physiology, 118(11), 2477-2487. https://doi.org/10.1007/s00421-018-3974-0

Taylor, I. M., Boat, R., \& Murphy, S. L. (2018). Integrating theories of self-control and motivation to advance endurance performance. International Review of Sport and Exercise Psychology, $0(0)$, 1-20. https://doi.org/10.1080/1750984X.2018.1480050

Terry, P. C., Lane, A. M., \& Fogarty, G. J. (2003). Construct validity of the Profile of Mood StatesAdolescents for use with adults. Psychology of Sport and Exercise, 4(2), 125-139. https://doi.org/10.1016/S1469-0292(01)00035-8

Vadillo, M. A. (2019). Ego Depletion May Disappear by 2020. Social Psychology, 50(5-6), 282-291. https://doi.org/10.1027/1864-9335/a000375

Van Cutsem, J., Marcora, S., Pauw, K. D., Bailey, S., Meeusen, R., \& Roelands, B. (2017). The Effects of Mental Fatigue on Physical Performance: A Systematic Review. Sports Medicine, 47(8), 
1569-1588. https://doi.org/10.1007/s40279-016-0672-0

Vickers, J. N., \& Williams, A. M. (2017). The Role of Mental Processes in Elite Sports Performance. Oxford Research Encyclopedia of Psychology. https://doi.org/10.1093/acrefore/9780190236557.013.161

Wang, C., Trongnetrpunya, A., Babu, I., Samuel, H., Ding, M., \& Kluger, B. M. (2016). Compensatory Neural Activity in Response to Cognitive Fatigue. 36(14), 3919-3924. https://doi.org/10.1523/JNEUROSCI.3652-15.2016

Wiemers, E. A., \& Redick, T. S. (2018). Working memory capacity and intra-individual variability of proactive control. Acta Psychologica, 182, 21-31. https://doi.org/10.1016/j.actpsy.2017.11.002 